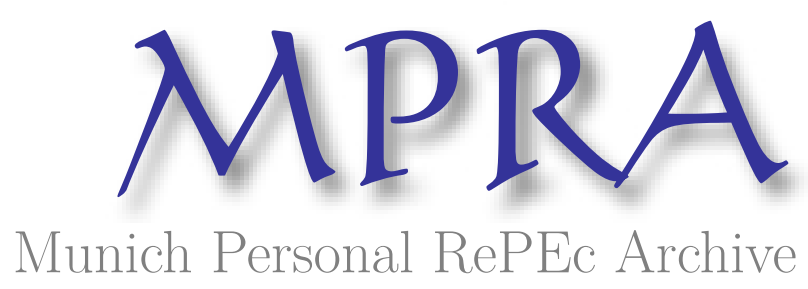

\title{
The perils of first-order conditions of New Keynesian models
}

Kim, Minseong

14 April 2016

Online at https://mpra.ub.uni-muenchen.de/70753/

MPRA Paper No. 70753, posted 15 Apr 2016 07:23 UTC 


\title{
The perils of first-order conditions of New Keynesian models
}

\author{
Minseong Kim
}

$2016 / 04 / 14$

\begin{abstract}
For deriving equilibrium of sticky-price/monopolistic competition New Keynesian models, first-order conditions are often used. This paper shows that they may not be sufficient and presents a case out of a simple model where there exists no equilibrium. This is true even when Taylor rule is assumed.
\end{abstract}

\section{Modified Rotemberg sticky-price model}

In Kim (2016) [3], I discussed how a Rotemberg model [5] leads to accounting inconsistency problems. But there exists modification [4] of the Rotemberg model, where the firm pays the inflation costs to the household.

The household has the following optimization problem:

$$
\max _{C_{t}, B_{t}, N_{t}} U\left(C_{t}, N_{t}\right)=\sum_{t=0}^{\infty} \beta^{t}\left[\frac{C_{t}^{1-\sigma}}{1-\sigma}-\frac{N_{t}^{1+\varphi}}{1+\varphi}\right]
$$

with the modified budget constraint:

$$
P_{t} C_{t}+\frac{B_{t}}{R_{t}} \leq W_{t} N_{t}+D_{t}+B_{t-1}+P_{t} \frac{\chi}{2}\left(\frac{P_{t}}{P_{t-1}}-1\right)^{2} C_{t}
$$

where $P_{t}$ refers to price level, $B_{t}$ refers to a one-time bond, $C_{t}$ refers to consumption, $W_{t}$ refers to nominal wage, $N_{t}$ is labor, $D_{t}$ is profit dividends receives from a continuum of firms.

$\chi$ is the degree of price rigidity, and this form allows $C_{t}=Y_{t}$ in equilibrium, unlike the standard Rotemberg model.

Notice the additional payment made by the firm to the household. One could have assumed that this payment is given to the household optimization problem rather than being dependent on $C_{t}$, which means that the household has the control over this payment. But doing so makes less sense, since the payment made by the firm is due to some service/household products relating to inflation/deflation. 
I will only derive the optimality condition for labor/consumption, since the IS equation is not relevant for rest of this paper.

$$
\frac{W_{t}}{P_{t}}=C_{t}{ }^{\sigma} N_{t}^{\varphi}\left[1-\frac{\chi}{2}\left(\frac{P_{t}}{P_{t-1}}-1\right)^{2}\right]=u_{t} C_{t}{ }^{\sigma} N_{t}^{\varphi}
$$

One immediately sees one problem with this specification: $u_{t}$ can go negative for high inflation, which means negative real wage. I will restrict to $0<u_{t} \leq 1$. For simplification, I will set $P_{0}=1$.

Let there be a continuum of firms $[0,1]$. The production function of each firm is:

$$
C_{t}(i)=A_{t} N_{t}(i)
$$

Aggregating over,

$$
C_{t}=s_{t} A_{t} N_{t}
$$

where $0<s_{t} \leq 1$, which is inverse price dispersion factor. In this modified Rotemberg model, $s_{t}=1$.

Let the classical equilibrium at time $t=0$ be $C_{e}, N_{e}$. Since $W_{e} N_{e}=C_{e}$ in the classical equilibrium with $W_{e}=C_{e}{ }^{\sigma} N_{e}^{\varphi}$,

$$
\begin{gathered}
C_{e}=W_{e} N_{e}=C_{e}{ }^{\sigma} N_{e}{ }^{\varphi+1}=\frac{C_{e}^{1+\sigma+\varphi}}{A_{0}^{1+\varphi}} \\
C_{e}=A_{e}{ }^{\frac{1+\varphi}{\sigma+\varphi}}
\end{gathered}
$$

Let the sticky-price monopolistic-competition equilibrium be $C_{0}, N_{0}$ at $t=0$. Relating $C_{0}$ and $C_{e}$ by $C_{0}=k C_{e}$,

$$
\begin{gathered}
D_{0}=C_{0}-W_{0} N_{0}=k C_{e}-u_{0} \frac{k^{1+\sigma+\varphi} C_{e}{ }^{1+\sigma+\varphi}}{A_{0}^{1+\varphi}} \\
W_{0} N_{e}=u_{0} \frac{k^{\sigma+\varphi} C_{e}{ }^{\sigma+\varphi}}{A_{0}{ }^{1+\varphi}} \frac{C_{e}}{A_{0}}=u_{0} \frac{k^{\sigma+\varphi} C_{e}{ }^{1+\sigma+\varphi}}{A_{0}{ }^{1+\varphi}} \\
W_{0} N_{e}+D_{0}=k C_{e}-u_{0} \frac{k^{1+\sigma+\varphi} C_{e}{ }^{1+\sigma+\varphi}}{A_{0}^{1+\varphi}}+u_{0} \frac{k^{\sigma+\varphi} C_{e}{ }^{1+\sigma+\varphi}}{A_{0}{ }^{1+\varphi}}
\end{gathered}
$$

The idea is that for the household problem $W_{0}$ and $N_{0}$ are given. If the household wants $C_{e}$ and $N_{e}$ as an outcome, would this satisfy the budget constraint? And if so, would $\left(C_{e}, N_{e}\right)$ be preferred over $\left(C_{0}, N_{0}\right)$ ? First, it seems that the answer to the latter question be no, because the first-order optimality condition is not satisfied by $\left(C_{e}, N_{e}\right)$. But what this paper will show is that the condition is not sufficient. After all, the FOC is only the necessary condition.

$$
\begin{gathered}
k C_{e}-u_{0} \frac{k^{1+\sigma+\varphi} C_{e}^{1+\sigma+\varphi}}{A_{0}{ }^{1+\varphi}}+u_{0} \frac{k^{\sigma+\varphi} C_{e}{ }^{1+\sigma+\varphi}}{A_{0}{ }^{1+\varphi}} \geq C_{e} \\
C_{e}{ }^{1+\sigma+\varphi} u_{0}\left[k^{\sigma+\varphi}-k^{1+\sigma+\varphi}\right] \geq(1-k) A_{0}{ }^{1+\varphi} C_{e}
\end{gathered}
$$




$$
u_{0} k^{\sigma+\varphi}[1-k] \geq 1-k
$$

For $k \geq 1$,

$$
u_{0} k^{\sigma+\varphi} \leq 1
$$

It is easy to find $u_{0}$ that satisfies Equation 14 given $k$. For a welfare evaluation example, suppose $A_{0}=1$. Then, $C_{e}=1$. Let $k=2$, which means $C_{0}=2$, with $N_{0}=2$. Let $\sigma=0.5, \varphi=0.5$.

Utility provided by $\left(C_{0}, N_{0}\right)$ is $w\left(C_{0}, N_{0}\right)=w(2,2)=2^{0} .5 / 0.5-2^{1} .5 / 1.5 \approx$ 0.943. $w\left(C_{e}, N_{e}\right)=1 / 0.5-1 / 1.5 \approx 1.333$. Thus, $w\left(C_{e}, N_{e}\right)>w\left(C_{0}, N_{0}\right)$.

With $u=1 / 2$, Equation 14 is satisfied and $0<u \leq 1$ is retained, with inflation of $100 \%$ when $\chi=1$.

What this welfare calibration shows is that this Rotemberg model inevitably leads to disequilibrium for some sets of inflation and output that were previously thought as possible equilibria. The household, given $W_{t}, P_{t}, D_{t}, B_{-1}$, will prefer $\left(C_{e}, N_{e}\right)$ over $\left(C_{0}, N_{0}\right)$ and thus will not choose $\left(C_{0}, N_{0}\right)$. This demonstrates the fact that FOC is only a necessary one, but does not guarantee that the correct solution would be achieved.

An additional interpretation of Equation 14 would be that for each $k>1, u_{0}$ must be greater than a certain point in order to be considered as a possible equilibrium - meaning that inflation rate cannot go below or above a certain level. For $k<1$, equation 13 is not satisfied automatically.

\subsection{Output asymmetry and monetary policy}

The above discussion shows that whenever the output is higher than the classical level, disequilibrium is the only possible result for non-zero inflation. Even if one adjusts $u_{t}$ to rule out $\left(C_{e}, N_{e}\right)$, there always exists a nearby result $(C, N)$ that is better relative to the original $\left(C_{t}, N_{t}\right)$. This continues until $u_{t}=1$, which rules out inflation. Getting the cue from the New Keynesian Phillips Curve (NKPC), which can also be derived from the first-order approximation solution of the Rotemberg model around zero inflation steady state:

$$
\pi_{t} \equiv \frac{P_{t}}{P_{t-1}}-1=\beta E_{t}\left[\pi_{t+1}\right]+\kappa \tilde{y}_{t}
$$

where $c_{t}=y_{t}=\log \left(Y_{t}\right)$ and $\tilde{y}_{t}=\log \left(Y_{t} / Y_{n, t}\right)$ (output gap) with $Y_{n, t}$ the natural level obtained without sticky price but with monopolistic competition. $\kappa>0$ is assumed. Thus, down to some positive output gap (because $Y_{n, 0}<Y_{e}$ ), $\pi_{t}$ is constrained to zero.

$$
\beta E_{t}\left[\pi_{t+1}\right]=-\kappa \tilde{y_{t}}
$$

This suggests that when $\tilde{y}_{t}$ is above the classical level, one must expect deflation at the next period.

To the first order approximation around zero inflation steady state with the value of $\chi$ small enough, one can also recover the standard IS equation:

$$
E_{t}\left[c_{t+1}\right]=c_{t}+\frac{1}{\sigma}\left(i_{t}-E_{t}\left[\pi_{t+1}\right]-\rho\right)
$$


where $\rho=-\log \beta, c_{t}=\log C_{t}$ with $i_{t}$ being nominal interest rate. Reformulating as the output gap version,

$$
E_{t}\left[\tilde{c_{t+1}}\right]=\tilde{c_{t}}+\frac{1}{\sigma}\left(i_{t}-E_{t}\left[\pi_{t+1}\right]-r_{t}^{n}\right)
$$

where $r_{t}^{n}$ is the natural rate of interest.

Thus, if $y_{t}$ is greater than the classical level $y_{e}, i_{t} \leq r_{t}^{n}-\left(y_{t}-y_{e}\right)$ must be satisfied to retain equilibrium, assuming technology remains constant - otherwise, inflation expectation would be inconsistent. Thus, any boom would instantaneously be curbed - implying output asymmetry. If central bank has control on $i_{t}$ and sets it wrong, then disequilibrium is inevitable.

\section{Calvo model}

The Calvo sticky-price model [1] presented here has the same household optimization problem as before except that the budget constraint is now

$$
P_{t} C_{t}+\frac{B_{t}}{R_{t}} \leq W_{t} N_{t}+D_{t}+B_{t-1}
$$

Thus real wage first-order condition is given by

$$
\frac{W_{t}}{P_{t}}=C_{t}^{\sigma} N_{t}^{\varphi}
$$

Again assume $P_{0}=1$.

In the Calvo model, production function for an individual firm remains the same, but now price dispersion affects the final output. Production function will be changed to:

$$
Y_{t}=s_{t} A_{t} N_{t}^{1-\alpha}
$$

For the Calvo model, $0<s_{t} \leq 1$, and no one value can be pre-determined for $s_{t}$ without specified monetary policy.

Decreasing returns to scale is required for what follows - in constant returns to scale, the method presented below would fail, as will be seen.

I will assume $P_{e}=1$.

$$
\begin{gathered}
C_{e}=W_{e} N_{e}=\frac{C_{e}{ }^{\sigma+\frac{1+\varphi}{1-\alpha}}}{A_{0}^{\frac{1+\varphi}{1-\alpha}}} \\
C_{e}=A_{0}^{\frac{1+\varphi}{\sigma-\alpha \sigma+\varphi+\alpha}} \\
N_{e}=A_{0}^{\frac{1+\varphi-\sigma+\alpha \sigma-\varphi-\alpha}{(\sigma-\alpha \sigma+\varphi+\alpha)(1-\alpha)}} \\
D_{0}=A_{0} \frac{1+\varphi}{\sigma-\alpha \sigma+\varphi+\alpha}\left[k-\frac{k^{\frac{\sigma-\alpha \sigma+1+\varphi}{1-\alpha}}}{s_{0}^{\frac{1+\varphi}{1-\alpha}}}\right] \\
W_{0} N_{e}=A_{0}^{\frac{1+\varphi}{\sigma-\alpha \sigma+\varphi+\alpha}} \frac{k^{\frac{\sigma-\alpha \sigma+\varphi}{1-\alpha}}}{s_{0}^{\frac{\varphi}{1-\alpha}}}
\end{gathered}
$$




$$
W_{0} N_{e}+D_{0}=A_{0}^{\frac{1+\varphi}{\sigma-\alpha \sigma+\varphi+\alpha}}\left[\frac{k^{\frac{\sigma-\alpha \sigma+\varphi}{1-\alpha}}}{s_{0}^{\frac{\varphi}{1-\alpha}}}+k-\frac{k^{\frac{\sigma-\alpha \sigma+1+\varphi}{1-\alpha}}}{s_{0}^{\frac{1+\varphi}{1-\alpha}}}\right]
$$

It is wished that $C_{e} \leq W_{0} N_{e}+D_{0}$, thus:

$$
1 \leq\left[\frac{k^{\frac{\sigma-\alpha \sigma+\varphi}{1-\alpha}}}{s_{0}^{\frac{\varphi}{1-\alpha}}}+k-\frac{k^{\frac{\sigma-\alpha \sigma+1+\varphi}{1-\alpha}}}{s_{0}^{\frac{1+\varphi}{1-\alpha}}}\right]
$$

For calibration, suppose that $s_{0}$ is very close to 1 that $s_{0}=1$ can be assumed. Then,

$$
1 \leq\left[k^{\frac{\sigma-\alpha \sigma+\varphi}{1-\alpha}}+k-k^{\frac{\sigma-\alpha \sigma+1+\varphi}{1-\alpha}}\right]
$$

Re-arranging,

$$
1-k \leq k^{\frac{\sigma-\alpha \sigma+\varphi}{1-\alpha}}\left[1-k^{\frac{1}{1-\alpha}}\right]
$$

Let $k<1$.

Again, I will assume that $0<\alpha<1$ is very close to 1 , but not 1 , and let $0<\varphi$ be close to 0 , but not 0 . Similarly let $0<\sigma$ be close to 0 but not 0 . Then the RHS approaches 1 as these parameters become close to the assigned limits, with $\varphi$ coming close to 0 faster than $1-\alpha$.

This demonstrates that there exists some $(\alpha, \sigma, \varphi, k)$ that satisfies Equation 30, but it can easily be shown that in case of constant returns to scale $(\alpha=0)$, Equation 30 does not hold.

This existence proof demonstrates that some $\left(C_{0}, N_{0}\right)$ that is supposed to be some equilibrium of the Calvo model via first-order conditions, is not actually an equilibrium, since the household definitely prefers $\left(C_{e}, N_{e}\right)$ over $\left(C_{0}, N_{0}\right)$.

\section{Conclusion}

This paper suggests that first-order conditions derived from optimization problems are not sufficient to find a sticky-price monopolistic competition (Rotemberg, Calvo) model equilibrium, unless one moves away from standard interpretations. The original Rotemberg model suffers from the accounting problem exposed in [3], and thus this paper uses the variant for demonstration. Interpretation of New Keynesian Phillips Curve may have to change - inflation is locked to zero for a range of outputs.

For the Calvo model, while not discussed in this paper, sub-utility function in terms of CES aggregator may be understood as a final good producer production function. Usually, it is assumed that by competitive assumptions zero profit results in for the final good producer. But this certainly does not have to be the case (zero profit is assumed, not derived). When the condition is relaxed, a different understanding can be reached. 


\section{References}

[1] Calvo, G. A. (1983). "Staggered prices in a utility-maximizing framework", Journal of Monetary Economics 12 (3): 383-398.

[2] Gali, J. (2014). "The Effects of a Money-Financed Fiscal Stimulus", CEPR Discussion Paper 10165, September.

[3] Kim, M. (2016). "How accounting accuracy affects DSGE models", Preprint.

[4] Moll, B. (2012). "New Keynesian Model in Continuous Time", Slides.

[5] Rotemberg, J. (1982). "Sticky Prices in the United States", Journal of Political Economy 90 (6): 1187-1211. 